\title{
Sequence Tolerant Segmentation System of Brain MRI
}

\author{
Yuhua Gu, Lawrence O. Hall, Dmitry Goldgof, Parag M. Kanade, F. Reed Murtagh ${ }^{1}$ \\ Dept. of Computer Science \& Engineering, ${ }^{1}$ College of Medicine \\ University of South Florida, Tampa, FL 33620, U.S.A. \\ hall@cse.usf.edu
}

\begin{abstract}
An automatic human brain segmentation system for magnetic resonance images is presented. It has two main parts: a fuzzy clustering algorithm and a set of cluster combination rules. Images are segmented into ten classes by the unsupervised fuzzy c-means clustering algorithm. Then a knowledge-based system labels the clusters into the tissues of interest: cerebrospinal fluid, gray matter and white matter. This approach can process MRI data that comes from different scanners with different sequences and head coils, using several different spin-echo images (with different echo times) and different slice thickness. The system adapts without manual intervention. Segmented synthetic image data from the brainweb Simulated Normal Brain Database resulted in a one voxel away accuracy of $90 \%$. The results from real data from various magnetic resonance imagers were compared with a radiologist's segmentation and found to generally agree within 10\%, the typical range of interrater radiologist agreement.
\end{abstract}

Keywords: MRI, fuzzy c-means, knowledge-based system, brain segmentation, CSF, gray matter, white matter.

\section{Introduction}

Magnetic resonance (MR) imaging uses magnetization and radio waves, rather than x-rays to make very detailed, cross-sectional pictures of the brain. Segmented brain images are used to visualize a volume and quantitatively analyze anatomical and functional cortical structures. Segmented brain tissues provide an anatomical framework for functional visualization, which has a potential use in neuroscience research and neurosurgical planning owing to advances in functional MRI (fMRI) [1]. Moreover, segmented brain images are widely used in cortical surface mapping, volume measurement, tissue classification and differentiation, functional and morphological adaptation assessment, and the characterization of neurological disorders such as multiple sclerosis, stroke, and Alzheimer's disease. MRI brain segmentation has played a critical role in these technical advancements.

For any segmentation used in clinical applications, it is important that it be of high precision and accuracy. There are many conventional methods of MRI segmentation that use image processing techniques such as region growing, edge detection, histogram based approaches [2], etc. The problem with all these methods is that, they need human interaction for accurate and reliable segmentation. This is usually in the form of a small amount of labeled data for some or all classes, which is usually time-consuming and expensive (requiring physician expertise for the initial knowledge). Many approaches to image segmentation try to segment regions containing voxels with similar intensity values. However, intensity inconsistencies which are caused by the effect of the inhomogeneity of medical imaging modalities and biological variations of tissues become the main obstacles for the segmentation. To solve this problem, many methods, such as statistical, structural and knowledgebased have been proposed during last 10 years [3], [4], [5], [6], [7], [8].

Many automatic segmentation methods for medical images have been developed [2], [8], [9], [10], [16], [17], [18], [19], [20], and they all have very good performance. The goal of the automatic system is to make diagnosis quick and effective. However, some of the automatic systems still require human interaction. The automatic system described in this paper is an attempt to provide completely automatic segmentation and labeling of the tissues of interest such as cerebrospinal fluid (CSF), white matter and gray matter. The main advantage of our system over other automatic systems is sequence tolerance.

We process slices to take advantage of anatomical knowledge, which is invariant across users. In [10], a sequence tolerant system for segmenting the brain was proposed by M.S. Atkins, which has been successful in segmenting the brain in every slice from head images acquired from several different MRI scanners, using different resolution images and different echo sequences. However, the other work mentioned above cannot adapt to many sequences. Additionally, we have 3-Tesla data available. The different sequences of the data for our system are presented in Table 1. The method described here builds on previous work from [6], [7]. It was organized as following two steps: (1) Each slice (containing T1-weighted, Proton-Density weighted and T2-weighted images) in the volume was initially oversegmented into a set of classes with fuzzy memberships by an unsupervised fuzzy c-means algorithm (FCM) [11], [12]. (2) A knowledge-based system was used to automatically locate the main brain tissues. In the upcoming sections, our system will be described in detail and results presented. 


\section{Images}

The heads of volunteers were imaged in the transaxial plane, the expected orientation for our system. Twenty volumes of data were obtained from SIEMENS MRI machines at the University of Miami. Fifteen of them were from a 1.5Tesla magnetic field system. The other five of them were from a 3-Tesla magnetic field system. There is another 1.5Tesla system data set which came from a SIEMENS MRI machine at the University of California at San Francisco. Two volumes of synthetic magnetic resonance (MR) image data [15] whose ground truth were available have also been processed by the segmentation system. One volume has $0 \%$ gaussian noise and $0 \%$ intensity non-uniformity (Radio Frequency), the other one contains 3\% gaussian noise and $20 \%$ intensity non-uniformity. All the volumes consist of three modality images: T1-weighted (T1), Proton Density weighted (PD) and T2-weighted (T2). The different acquisition protocols for the above volumes are presented in Table 1. Based on the value of magnetic field strength, the data can be divided into two categories: 1.5-Tesla and 3-Tesla system data. The synthetic data has no definition of magnetic field strength at all, however, it contains a very similar cluster distribution to data from the other two 1.5Tesla systems. Moreover, the segmented results from the synthetic data indicated that it could be segmented by our 1.5-Tesla system.

Our knowledge-based system rules were constructed based on these two categories. We have different 1.5Tesla volumes with different protocol settings, no matter how different their protocols are, as long as they have the same magnetic field strength, the segmentation system could still adapt to those different volumes and produce good results. For 3-Tesla data, we have extracted some different knowledge-based rules. The difference in the system rules will be discussed in detail later. From Table 1, the slice thickness of $\mathrm{T} 1$ was either $1.5 \mathrm{~mm}$ or $1 \mathrm{~mm}$ with no interslice gap, however, T2 and PD slices have thickness of $3 \mathrm{~mm}$. Therefore, alignment needs to be performed by using registration software, which was provided by the $3 \mathrm{D}$ Volume Registration Viewer software [13]. All the images are spatially registered by the software. We can either do alignment towards a thicker slice thickness (take T2 as a reference image), or do alignment towards thinner slice thickness (take T1 as a reference image), in this case, more slices must be processed. All results reported here are from using $\mathrm{T} 2$ as the reference image.

\section{Methods}

The system developed was a modified version of that described in [7], which has shown that a combination of unsupervised fuzzy clustering and knowledge based techniques can efficiently segment and label brain tumors from an MRI slice of the brain. However, to adapt to the image data obtained from the SIEMENS MRI machine, the original system has been modified to allow the segmentation and
TABLE 1

\section{ACQUISITION PARAMETERS}

\begin{tabular}{|c|c|c|c|c|}
\hline Source & Modality & $\begin{array}{c}\text { Slice Thickness } \\
(\mathrm{mm})\end{array}$ & $\begin{array}{c}\text { TR } \\
(\mathrm{ms})\end{array}$ & $\begin{array}{c}\text { TE } \\
(\mathrm{ms})\end{array}$ \\
\hline \multirow{3}{*}{ UM (1.5-Tesla) } & T1 & $1.0 / 1.5$ & 1800 & 4.38 \\
\cline { 2 - 5 } & PD & 3.0 & 4600 & 20 \\
\cline { 2 - 5 } & T2 & 3.0 & 4600 & 80 \\
\hline \multirow{3}{*}{ UCSF (1.5-Tesla) } & T1 & 1.5 & 9.7 & 4.00 \\
\cline { 2 - 5 } & PD & 3.0 & 4000 & 30 \\
\cline { 2 - 5 } & T2 & 3.0 & 4000 & 80 \\
\hline \multirow{3}{*}{ Brain web } & T1 & 3.0 & - & - \\
\cline { 2 - 5 } & PD & 3.0 & - & - \\
\cline { 2 - 5 } & T2 & 3.0 & - & - \\
\hline \multirow{3}{*}{ UM (3-Tesla) } & T1 & 1.0 & 2150 & 4.38 \\
\cline { 2 - 5 } & PD & 3.0 & 4000 & 30 \\
\cline { 2 - 5 } & T2 & 3.0 & 4000 & 80 \\
\hline
\end{tabular}

labeling of normal MRI brain volumes into the tissues of interest. The volumes used in our work consist of more than 40 slices ( $3 \mathrm{~mm}$ thickness), beginning with the "center slice", which lies approximately 7 to $8 \mathrm{~cm}$ from the top of the head in transaxial plane. This slice is through the lateral ventricles and provides a good landmark for knowledgebased processing. From the center slice, segmentation proceeds to the other slices located towards the top of the head and through the bottom of the head. The reason that we selected this particular region for a center slice is that it has the most reliable anatomical information for the knowledge-based system rules. In the process of the FCM step, knowledge from a previous slice was used, so that cluster center values of the current slice were an initialization for the next slice. Clustering time was reduced and accuracy in processing the whole volume was improved by using knowledge propagation.

\subsection{Fuzzy c-means algorithm}

Fuzzy c-means (FCM) [11], [12] is an effective clustering algorithm, which rarely produces a very poor partition. The data used by FCM consists of T1 weighted, proton density weighted and T2 weighted images. T1-weighted images are acquired using a spin-echo sequence; the proton density and T2 weighted images are acquired using a fast spin echo sequence. A typical set of labels might be background (air), skull, skin (or fat), gray matter (GM), white matter (WM) and cerebrospinal fluid (CSF) and tumor (if applicable). The number of clusters can be set as large as we want, however, this will tend to result in some degree of over-segmentation in normal slices. The number of classes was set to be ten, which was found effective in [6]. The FCM algorithm steps are described as follows: let $\mathrm{c}$ be integer, $1 \leq c \leq n$ (number of clusters), the exponent $m>1$ ( $\mathrm{m}=2$ here) is used to adjust the weighting effect of the membership value, and let $X=x_{1}, x_{2}, \ldots, x_{n}$ be the set of d-dimensional ( $\mathrm{d}=3$ ) feature vectors, our purpose is to calculate $u_{j}=\left\{u_{1 j}, u_{2 j}, \ldots, u_{c j}\right\}$, which is the grade of membership at feature point $x_{j}$.

Because of the inhomogeneity of the MR image, some 
voxels of the same tissues may be assigned to different clusters, voxels in the same cluster may be assigned to different tissues and partial membership values are useful. After clustering one must find the correspondence between memberships and tissues.

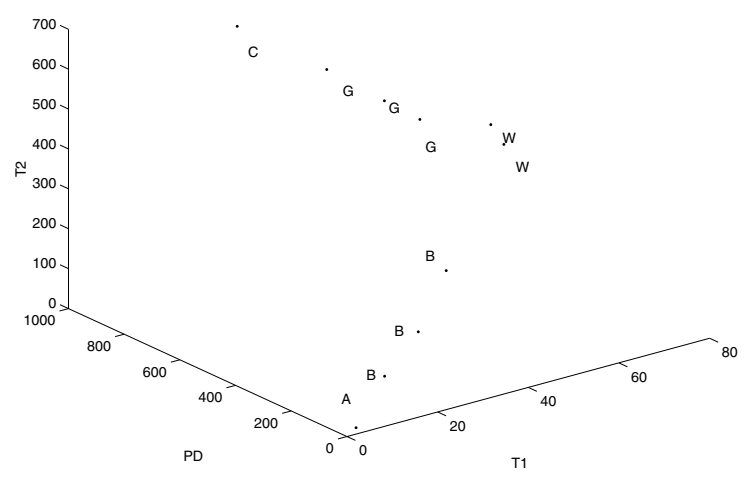

Fig. 1. Class centers in T1, PD and T2 space (1.5-Tesla)

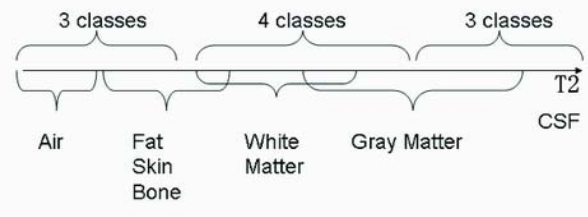

Fig. 2. T2 Spectrum (1.5-Tesla)

\subsection{Feature space knowledge}

The FCM clustering algorithm took a slice with three images as input and clustered it into ten classes. Each class has a cluster center (T1, PD and T2), Fig. 1 shows the ten cluster centers distributed in 3-D feature space for a typical partition. Different characters were used to represent classes of Air (A), skull tissues (B), white matter $(\mathrm{W})$, gray matter $(\mathrm{G})$, and CSF $(\mathrm{C})$, respectively. To better illustrate the cluster centers in feature space, only the T2 spectrum is shown in Fig. 2. These figures contain the information used in locating focus-of-attention tissues. The feature values of the images depend on the repetition time and echo time which were used in scanning, we not only have 1.5-Tesla image data (including the synthetic data from brainweb) but also have 3-Tesla data, which came from another SIEMENS machine. The distributions of the ten cluster centers in feature space exhibited totally different cluster distribution for the different field strengths. By visual examination of the figures (Fig. 1 vs Fig. 3), the following chunks of knowledge for 1.5 and 3-Tesla have been extracted, respectively. Especially, by comparing the T2 spectrum figures (Fig. 2 vs Fig. 4), we could see the cluster distribution for 3-Tesla images is more complicated than the 1.5-Tesla, here different rules have been created. 1.5-Tesla knowledge:

1. The air class is always in the lowest one or two classes in $\mathrm{T} 2$.

2. The two or three lowest $\mathrm{T} 2$ classes adjacent to the air class belong to skull tissues.

3. White matter was segmented into one or two classes. Its $\mathrm{T} 2$ centroid value is smaller than gray matter in $\mathrm{T} 2$, but higher than skull tissue classes.

4. Gray matter appears as one to three classes, the $\mathrm{T} 2$ value is smaller than CSF.

5. CSF always appears in the highest $\mathrm{T} 2$ class.

Since the tissue distribution obtained from the T2 spectrum (Fig. 4) had more overlap for 3-Tesla, we were unable to use the rules for 1.5-Tesla to deal with the 3-Tesla data. Additional information had to be extracted. We used the class distribution in T1 vs T2 space. Corresponding rules have been changed or added according to the new knowledge derived. The detailed knowledge extracted follows.

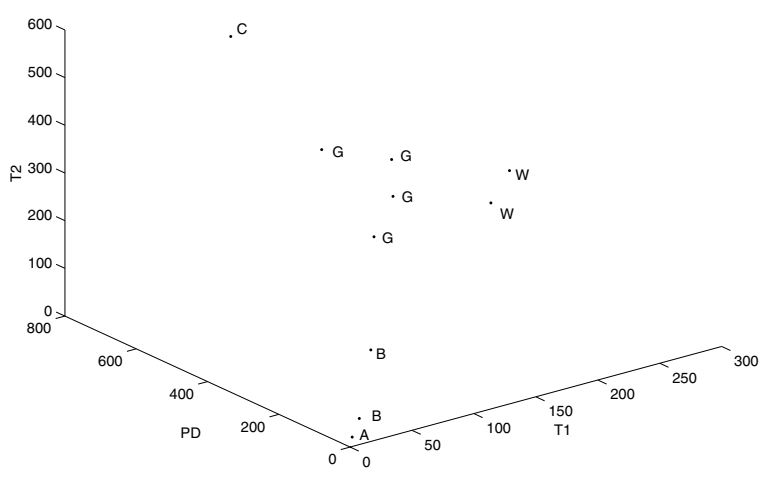

Fig. 3. Class centers in T1,PD and T2 space (3-Tesla)

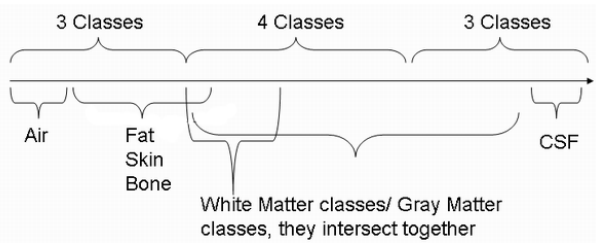

Fig. 4. T2 Spectrum (3-Tesla)

3-Tesla knowledge:

1. The air class is always in the lowest one or two classes in $\mathrm{T} 2$.

2. The two or three classes in $\mathrm{T} 2$ adjacent to the air class were skull tissues.

3. White matter may be clustered into one or two classes, the last class in $\mathrm{T} 1$ is known to be the white matter class, assign the second highest $\mathrm{T} 1$ class as a candidate white 


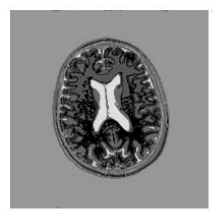

(a)

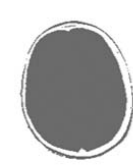

(b)

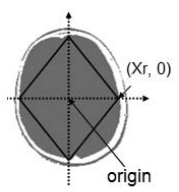

(c)
Fig. 5. (a) Initial 10 cluster images. (b) Binary image after setting the lowest three $\mathrm{T} 2$ classes to white, and remaining classes to gray matter. (c) A quadrangle overlaid on (b).

matter class.

4. CSF always appears in the highest $\mathrm{T} 2$ class.

5 . The rest of the classes belong to gray matter in $\mathrm{T} 2$ space. This observation of class center distribution for 3-Tesla images was based on 5 volumes of MR image data.

\subsection{Separating Skull Tissues}

Fig. 5a shows the combination of 10 cluster images obtained from FCM segmentation with different gray levels. Removing extra-cranial tissues was our first goal. Basically, air and skull tissues are always located in the first 3 T2 classes. Sometimes fuzzy clustering places skull tissues in white matter or gray matter classes (Fig. 7b), those skull voxels are very hard to remove. Because they have similar intensity values to white matter or gray matter. Here, using anatomical knowledge a binary image (Fig. 5b) was created, the background (white color) was from the combined lowest 3 classes in the T2 spectrum, the foreground (gray color) represents the rest of the 7 classes. It is for sure that the lowest 3 classes in the T2 spectrum belong to air and skull tissues, however, the lowest 4 th or 5 th class might be skull tissue too or not. Therefore, based on this binary image (Fig. 5b), a quadrangle [6], [7] was created inside of the brain (Fig. 5c). This quadrangle was used in further analysis, since we already know that the lowest 3 classes in the T2 Spectrum were air and skull tissues, the rest of the class images need to be checked by the following rule:

If (The number of the foreground voxels within the quadrangle is small)

Then (we define it as a skull class)

Else (It is a class of interest)

Typically, a skull class has from 0 to 200 voxels in the quadrangle area, the other class usually has more voxels in the area. It is very easy to differentiate skull tissue in the typical skull class. However, we sometimes have an abnormal case. In Fig. 6 the image shown was one of the ten classes of 3-Tesla data which were segmented by the FCM algorithm. The number of voxels in the quadrangle of this image class was quite small, which resulted in the class being assigned to skull tissues. Therefore a new general rule was created by setting a threshold value on the $\mathrm{T} 2$ value of the class centers, which were observed from five volumes of 3-Tesla data. The new rule for 3-Tesla data is

If (T2 value of the class center is less than $T$ )

Then (It is a skull class)

Else (It is a class of interest)

Extra-cranial clusters (Fig. 7a) and intra-cranial clusters (Fig. 7b) are divided by the above rule. There should be a minimum of three tissue classes in the intra-cranial group, one for CSF, white matter and gray matter respectively.

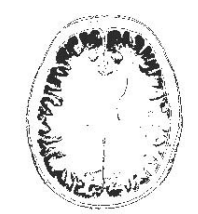

Fig. 6. Sample problem class from 3-Tesla

\subsection{Extracting the intra-cranial region}

As seen in Fig. 7b, there are still some "noise" tissues which belong to the extra-cranial group misplaced into the intra-cranial group during FCM clustering. Most of these noisy voxels are sparse spatially, however, some slices may contain large regions, such as the eyes, which we are not interested in and should be removed from further consideration. Li [6] used anatomical knowledge that extracranial tissues surround the brain and are not found within the brain itself. Therefore, a gross estimate of the brain must be created, then clusters consisting of extra-cranial tissues will have very few pixels inside this estimated brain. Our first important step here is to find the brain mask. The brain mask can be extracted by setting all intra-cranial clusters to foreground to create a binary mask, which is eroded with a $5 \times 5$ operator to remove small regions and enhance separation of the brain from surrounding tissues, then an eight-wise connected components operation was executed and the largest component was preserved, and other small components were set to be background. To recover the voxels lost during the erosion operation, a $5 \times 5$ dilation operator was applied on the new binary image. Some holes and voxels corresponding to intra-cranial clusters misplaced into extra-cranial clusters in the FCM step are filled after the dilation operation. An intra-cranial brain mask was created (Fig. 7c).

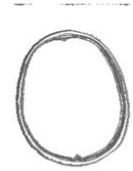

(a)

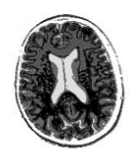

(b)

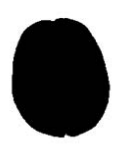

(c)
Fig. 7. (a) Extra-cranial clusters (b) intra-cranial clusters (c) intra-cranial brain mask 


\subsection{White matter splitting}

During initial FCM segmentation, we cluster each slice by clustering into more classes than tissue types [6]. Overclustering reduces the probability and degree of mixed classes [6], but also results in over segmentation of tissues of interest. One tissue may be split into two or three clusters. Tissue splitting will cause misclassification. In this section, white matter splitting detection techniques are discussed. Fig. 8 (a) and (b) show a white matter class and its neighbor (in feature space) white matter class, Fig. 8(c) and (d) are a white matter class and its neighbor class of gray matter, which was declared a candidate white matter class.

A two-level decision tree [6] was used to detect white matter splitting, the first level decision tree includes 3 components: Bi-orthogonal thickness (BT), a cluster density measure Density and adds additional knowledge from PDweighted space, which is a modified version from [7]. The BT method gives a coarse estimate of a component's size. In $\mathrm{Li}$ [6], it is declared that the BT of gray matter was never much larger than the BT of white matter. A class Density is defined as $\frac{P_{e}}{P_{o}}$, where $P_{e}$ represents the number of voxels in the cluster after erosion (ex: 5 x 5), $P_{o}$ represents the number of voxels in the original cluster. The known white matter cluster and candidate cluster can have a density value calculated, as an experimental result [6], the density of gray matter is less than white matter. White matter splitting can also be detected by using the distribution of cluster centers in feature space. If a candidate cluster is split white matter, the PD value of its cluster center is much closer to the known white matter class than a candidate which is gray matter. A rule can be built based on the three methods [7]. Another level of decision tree is shape analysis used to verify white matter splitting which was tentatively identified from the first level of the decision tree. Both of the candidate clusters from Fig. 8 have passed the first level rule, Fig. 9 (a) and (b) are the merged known cluster and candidate cluster after removing "noise" (by simply applying the "AND" operation between the class image and intra-cranial mask, because the noisy voxels are not in the range of the mask), however, one of them is false white matter splitting, which means one gray matter cluster has been tentatively assigned to white matter tissue. Because of the gray matter surrounding the white matter spatially, it will more likely be on the periphery in the intra-cranial mask. An intra-cranial ring can be obtained from the intracranial mask by applying the erosion operator $(7 \times 7)$ and subtracting the resultant image from original mask image. Fig. 9(c) and (d) show the rings obtained from the intracranial mask. A new ring (Fig. 9 (e) and (f) ) is generated by subtracting the merged image from the original ring. The number of voxels left in the second ring divided by the number of voxels in the original ring provide a ratio. The ratio is used to confirm whether a slice has white matter splitting, from Li's effort [6], the ratio of threshold value is 0.39 , white matter splitting will usually result in a ratio above 0.39 , while a slice without white matter splitting will have a ratio smaller than 0.36 .

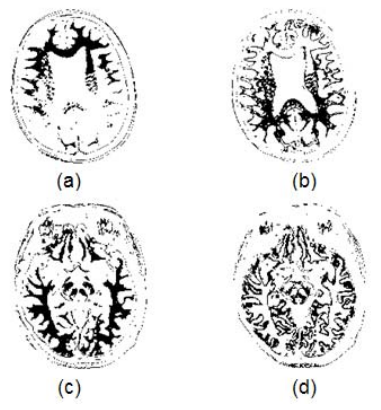

Fig. 8. The neighboring classes in two slices (a) A white matter class, (b) the neighboring white matter class of (a), (c) a white matter class, (d) the neighboring gray matter class of (c).

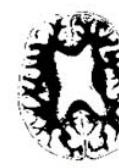

(a)

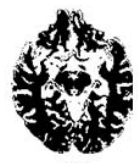

(b)

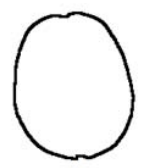

(c)

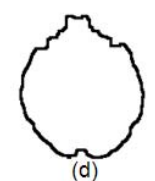

(d)

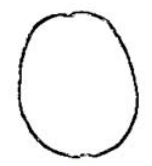

(e)

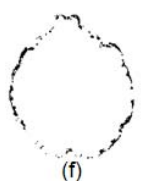

Fig. 9. Detect white matter splitting by using shape analysis: (a) Merged image of Fig 8 (a) and (b), (b) merged image of Fig 8 (c) and (d), (c) original ring of (a), (d) original ring of (b), (e) subtracting (a) from (c), (f) subtracting (b) from (d)

\subsection{CSF and Gray matter labeling}

CSF is easier to identify in normal brain data, as it has the highest T2 value class in feature space. Gray matter can be located after white matter and CSF are labeled, as the union of remaining clusters in intra-cranial group.

\section{Knowledge-based rules}

In this section, the difference in rules between 1.5-Tesla and 3-Tesla system will be highlighted. The differences were derived from the knowledge we obtained from the cluster distribution and some heuristic knowledge through observation.

Rule (1): compare-clusters-in-quadrangle-and-count-voxels Rule (2): load-sort-group2-cluster-in-T1-space (3-T)

Rule (3): remove-known-white-matter

Rule (4): assert-candidate-wm

Rule (5): assert-known-gray

Rule (1) is to extract the intra-cranial clusters. For the 3-Tesla version, in order to deal with the special case (ex: Fig. 6), the condition shown below was added. 

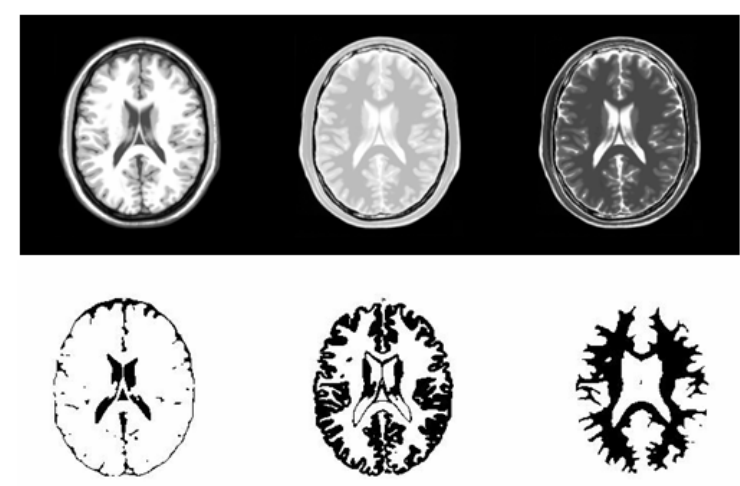

Fig. 10. Slice through the ventricle from synthetic data without noise (Synthetic 1 in Table 2): Top row are raw images, T1 weighted, PD weighted and T2 weighted images respectively, bottom row is the segmented CSF, GM and WM images, respectively
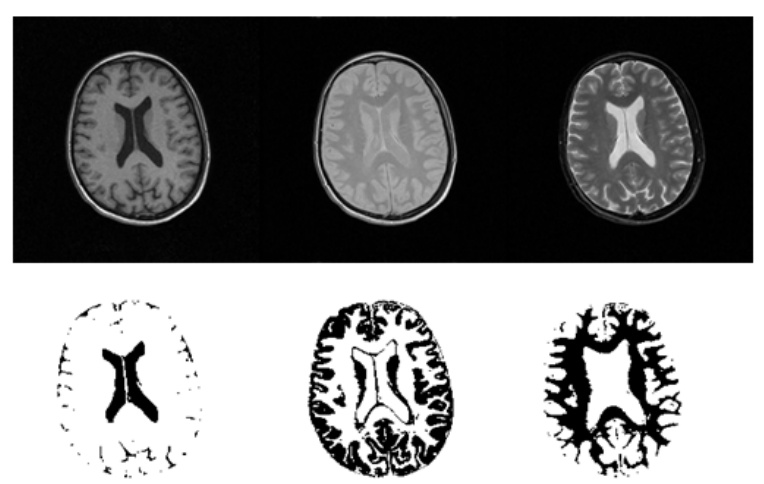

Fig. 11. Slice through the ventricle from 1.5-Tesla data (UM): Top row are raw images, T1 weighted, PD weighted and T2 weighted images respectively, bottom row is the segmented CSF, GM and WM images, respectively

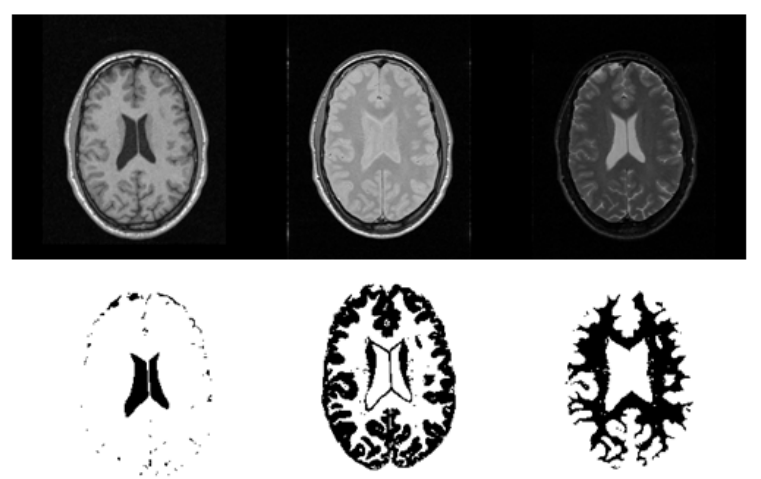

Fig. 12. Slice through the ventricle from 1.5-Tesla data (UCSF): Top row is the raw images, T1 weighted, PD weighted and T2 weighted images respectively, bottom row is the segmented CSF, GM and WM images, respectively
The class in Fig. 6 has very few voxels falling into the quadrangle, the rule for 1.5-Tesla failed to pass this class into the intra-cranial group. The 3-T addition is: If (The T2 value of the class center is less than $T$ )

Then (It is a skull class)

Else (It is a class of interest)

Here we set $\mathrm{T}$ to be 140 , this was empirically found after checking five volumes of 3-Tesla data. Rules (2)-(5) are the preliminary step for white matter splitting detection. Rules (3)-(5) are also in the 1.5-Tesla system, but they were changed for the 3-Tesla system. Rule (2) was added to order the cluster centers in $\mathrm{T} 1$ space. The essential differences can be described as follows:

Known WM:

1.5-T: The lowest $\mathrm{T} 2$ value class of the intra-cranial group 3-T: The highest T1 value class of the intra-cranial group Candidate WM:

1.5-T: The class in T2 space that is next to the known WM class in the intra-cranial group

3-T: The class that has the second highest $\mathrm{T} 1$ value in the intra-cranial group

Known GM:

1.5-T: The class in $\mathrm{T} 2$ space that is next to the candidate WM class in the intra-cranial group

3-T: The lowest T2 value class of the intra-cranial group which excludes the known GM class and the candidate WM class.

After finding the right known WM class, the candidate WM class and the known GM class, the other rules are kept unchanged, for example, white matter splitting detection rules still work as described in Section 3.5.

\section{Results}

We begin with a discussion of the two volumes of synthetic brain image data from brainweb which were segmented by our system. The volumes consist of fifty-nine slices with $256 \times 256$ voxels per slice, the thickness of each slice was $3 \mathrm{~mm}$. One volume has $0 \%$ gaussian noise and $0 \%$ intensity non-uniformity (Radio Frequency) (Synthetic 1 in Table 2), the other one has 3\% gaussian noise and $20 \%$ intensity non-uniformity (Synthetic 2 in Table 2). We chose the initial slice as a slice closest to the center of the head in the axial plane, where the ventricles are clearly visible in the images (Fig. 10). Segmentation is then extended to the other slices as described in Section 2. After sending these two volumes through the system, except some very low and very top slices, each slice was well segmented into $\mathrm{CSF}$, gray matter and white matter tissues. The synthetic data results were evaluated by comparing to ground truth images which are provided from brainweb. Our system performed well as indicated by the matching percentage for each tissue. The average accuracy was above $90 \%$ for most of the slices (center slice $+/-10$ slices). We did have some slices where accuracy wasn't higher than $90 \%$, when the 
labeling of white matter and gray matter was imperfect. The reason was that gray matter and white matter are close to each other in intensity on the boundary. The results for the synthetic data with noise and non-uniformity were not as good as expected. The percentage for CSF matching was not very high for some slices. The average accuracy for CSF matching was around $85 \%$. For GM and WM the accuracies were still above $90 \%$. Because the data had $20 \%$ intensity non-uniformity and $3 \%$ gaussian noise, inevitably there was an increase in inter-tissue overlapping.

Fig. 11 and Fig. 12 are typical segmented results for 1.5Tesla (real) data from UM and UCSF, respectively. The segmented results appear to be very good. We have two volumes of 1.5-Tesla data from UM which have ground truth images produced by a radiologist, unfortunately, each of them only contain 7 slices of ground truth. This included Fig. 11. The resulting segmentation had very good accuracy. The average accuracy of 7 slices was above 90\% (1.5T-UM 1 in Table 2). However, another 1.5-Tesla volume which has ground truth had poorer results (1.5T-UM 2 in Table 2). Fig. 13 is a example of that volume, obviously, this slice has CSF and gray matter mixed, there are too many CSF voxels in the segmented result, gray matter also looks too thin. The other results for the rest of slices for this volume are similar. It had average accuracy below $80 \%$ for CSF matching, average accuracy for GM was only $83.26 \%$. The result for 3-Tesla data was also very good visually, one slice is presented in Fig. 14, though we don't have ground truth images for 3-Tesla, visual results for 3-Tesla are acceptable.

TABLE 2

RESULTS

\begin{tabular}{|c|c|c|c|}
\hline Volume & CSF (Ave) & GM (Ave) & WM (Ave) \\
\hline Synthetic 1 (21 slices) & $92.50 \%$ & $96.18 \%$ & $95.09 \%$ \\
\hline Synthetic 2 (21 slices) & $85.35 \%$ & $92.75 \%$ & $92.27 \%$ \\
\hline 1.5T-UM 1 (7 slices) & $91.34 \%$ & $94.25 \%$ & $92.14 \%$ \\
\hline 1.5T-UM 2 (7 slices) & $76.80 \%$ & $83.26 \%$ & $87.32 \%$ \\
\hline
\end{tabular}

\section{Conclusions}

We have discussed a completely automatic segmentation system of MR Brain images. The system is easily adapted to different acquisition sequences as presented in Section 2, which is the main advantage of our method. However, since we have more data coming in the future, to make the system tolerant with sequences different from Table 1, we may need to capture new knowledge and tune the corresponding rules or parameters in the knowledge-based system. So far, our system works for 1.5-Tesla and 3-Tesla data. For most of the data we have now, good segmented results can be obtained, however, some slices are not so perfect, especially the voxels from the border between tissues. The problem came from the initial over-segmented FCM clustering. Clustering into more clusters than there are tissues allows for inhomogeneity to be successfully dealt with. A tissue type that is not homogeneous in a particular slice image will be put into different clusters which are then merged by rules which utilize anatomical and image space knowledge. Producing rules which are adaptable to different image acquisition characteristics remains an incompletely solved challenge. Certainly there are voxels which are made up of more than one tissue, which are a problem source. These voxels are on the border between tissues and our approach will tend to assign the voxels to the candidate class which is most characteristic. However, we have not carefully examined the accuracy on these voxels. We will focus on enhancing accuracy of segmentation of both systems (1.5-Tesla and 3-Tesla), making the system adapt to more sequences of 1.5-Tesla and 3-Tesla data, and moreover, make the system adapt to higher magnetic field strength data in the future, a goal is to have a menu of learnable MR and / or user settable parameters for new MR sequences.

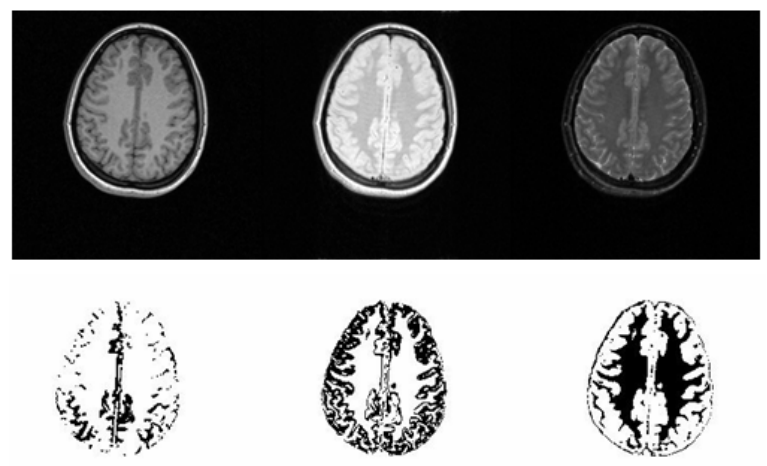

Fig. 13. Slice from 1.5 Tesla with a bad result (UM): Top row is the raw images, T1 weighted, PD weighted and T2 weighted images respectively, bottom row is the segmented CSF, GM and WM images, respectively
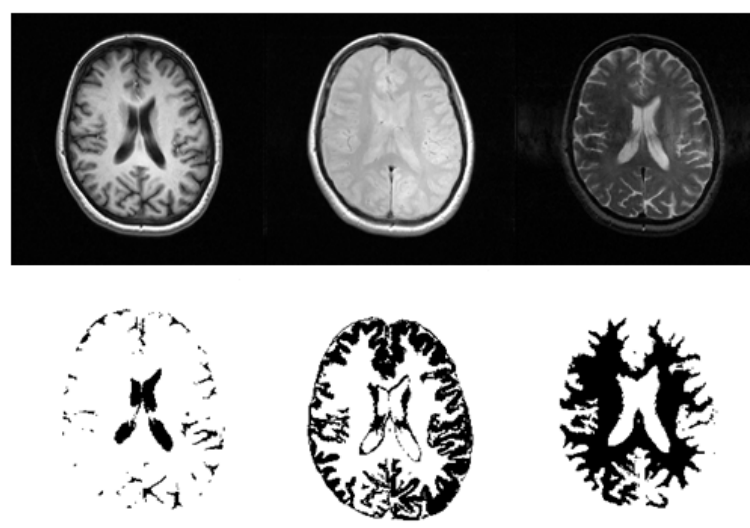

Fig. 14. Slice through the ventricle from 3-Tesla data (UM): Top row is the raw images, T1 weighted, PD weighted and T2 weighted images respectively, bottom row is the segmented CSF, GM and WM images, respectively 


\section{Acknowledgments}

This research partially supported by the National Institutes of Health under grant number 1 R01 EB00822-01.

\section{References}

[1] Functional MR Imaging (fMRI) - Brain: http://www.functionalmri.org/.

[2] Z.Y. Shan, G.H. Yue, J.Z. Liu, "Automated Histogram-Based Brain Segmentation in T1-Weighted Three-Dimensional Magnetic Resonance Head Images," NeuroImage v.17 pp. 1587-1598 2002.

[3] M.E. Brummer, R. M. Mersereau, R. L. Eisner, and R. R. J. Lewine, "Automatic detection of brain contours in MRI data sets," IEEE Trans. Med. Imag. vol. 12, no. 2, pp. 153-166, 1993.

[4] Z.Liang, R. J. Jaszczak, and R.E. Coleman, "Parameter estimation of finite mixtures using the EM algorithm and information criteria with application to medical image processing," IEEE Trans. Nucl. Sci. vol. 39, no. 4, pp. 1126-1133. 1992.

[5] M.E. Brandt, T.P. Bohan, L. A. Kramer, and J. M. Flecher, "Estimation of CSF, white and gray matter volumes in hydrocephalic children using fuzzy clustering of MR images," Computerized Med. Image. Graphics, vol. 18 , no.1., pp. 25-34,1994.

[6] C. Li, D. Goldgof, and L. Hall, "Knowledge-Based Classification and Tissue Labeling of MR Images of Human Brain," IEEE Trans. on Medical Imaging, vol. 12, no. 4, pp. 740-750, December 1993.

[7] M. Clark, L. Hall, D. Goldgof, R. Velthuizen, F. Murtagh, and M. Silbiger, "Automatic Tumor Segmentation Using Knowledge-Based Techniques," IEEE Trans on Medical Imaging, vol. 17, no. 2, pp. 187 201, April 1998.

[8] S. Zhang and J. Maeda, "A Rule-Based Expert System for Automatic Segmentation of Cerebral MRI Images," Proceedings of the 5th IEEE International Conference on Signal Processing, Beijing, pp. 21332138, 2000

[9] J. Alirezaie, M. E. Jernigan and C. Nahmias, "Automatic segmentation of Cerebral MR images using Artificial Neural Networks," IEEE Trans on Nuclear Sciences, vol. 45, no. 4, August 1998.
[10] M.S. Atkins and B.T. Mackiewich. "Fully automatic segmentation of the brain in MRI," IEEE Trans, on Medical Imaging, 17(1): 98-107, 1998.

[11] Cannon, RL, Dave, JV and JC Bezdek, "Efficient implementation of the fuzzy c-means clustering algorithms," IEEE Transaction on Pattern Analysis and Machine Intelligence, Vol. PAMI-8, No. 2,pp. 248-255, 1986.

[12] L. O. Hall, A. M. Bensaid, and et al, "A comparison of neural network and fuzzy clustering techniques in segmenting magnetic resonance images of the brain," IEEE transactions on Neural Networks, 3(5): 672-682, 1992.

[13] Colin Studholme, "3D Volume Registration Viewer," 1994-2004 http://www.colin-studholme.net/software/rview/rvmanual/rvman.htm.

[14] J.C. Bezdek, L.O. Hall, and L.P. Clarke, "Review of MR image segmentation techniques using pattern recognition," Medical Physics, 20(4): 1033-1048, 1993.

[15] Online simulated BrainWeb: http://www.bic.mni.mcgill.ca/brainweb/.

[16] Koen Van Leemput,* Frederik Maes, Dirk Vandermeulen, and Paul Suetens, "Automated Model-Based Tissue Classification of MR Images of the Brain," IEEE transactions on medical imaging, vol. 18, No. 10, Oct 1999.

[17] J. L. Marroquin, B. C. Vemuri*, S. Botello, "An Accurate and Efficient Bayesian Method for Automatic Segmentation of Brain MRI," IEEE transactions on medical imaging, vol. 21, No. 8, Aug 2002.

[18] M. Prastawa , E. Bullitt, N. Moon, K. Van Leemput, and G. Gerig, "Automatic Brain Tumor Segmentation by Subject Specific Modification of Atlas Priors." Academic Radiology, vol. 10, no. 12, pp. 1341-1348, Dec 2003.

[19] SM. Smith, "Fast robust automated brain extraction," Hum Brain Mapp, 17(3): 143-55, Nov 2002.

[20] CA. Cocosco, AP. Zijdenbos, AC. Evans. "A fully automatic and robust brain MRI tissue classification method," Med Image Anal, 7(4): 513-27, Dec 2003 